\title{
Pulmonic Valvuloplasty
}

National Cancer Institute

\section{Source}

National Cancer Institute. Pulmonic Valvuloplasty. NCI Thesaurus. Code C100044.

Dilation of the valve between the right ventricle and the pulmonary artery of the heart by inflating a balloon. (ACC) 